\title{
Trabalho de Grupo com Portadores de Ler/Dort: Relato de Experiência
}

\author{
Álvaro Roberto Crespo Merlo ${ }^{12}$ \\ Universidade Federal do Rio Grande do Sul \\ Maria da Graça Corrêa Jacques \\ Universidade Federal do Rio Grande do Sul \\ Maria da Graça Luderitz. Hoefel \\ Hospital de Clinicas de Porto Alegre
}

\begin{abstract}
Resumo
Neste artigo, apresenta-se e discute-se as contribuições da Psicologia ao estudo das Lesões por Esforços Repetitivos ou Distúrbios Osteomusculares Relacionados ao Trabalho (LER/DORT). Privilegia-se a análise do trabalho com grupos de portadores de tal patologia, prestado por uma equipe multidisciplinar do Hospital de Clínicas de Porto Alegre, em parceria com a Faculdade de Medicina e o Instituto de Psicologia da Universidade Federal do Rio Grande do Sul. Parte-se do pressuposto de que o estudo da relação entre saúde e trabalho constitui-se como importante espaço de atuação do psicólogo e que são necessárias produções textuais, a partir de relatos de experiências, que apresentem, sistematizem e fundamentem propostas de intervenção. As atividades com grupos, complementando os procedimentos terapêuticos convencionais, têm possibilitado que os portadores de LER/DORT possam diminuir sua culpabilização e reforçar sua independência e autonomia, minimizando, assim, o sofrimento psicológico associado à dor crônica e aos limites impostos pela doença.

Palavras-chave: Lesões por esforços repetitivos; doença ocupacional; trabalho com grupos.
\end{abstract}

\section{Groups Activity with Repetitive Strain Injuries Workers: Experience Report}

\begin{abstract}
This article presents and discusses the contributions of Psychology to the study of Repetitive Strain Injuries or Work Related Musculoskeletal Disorders. It privileges the analysis of the work carried out, jointly, by a multidisciplinary team of the Hospital de Clínicas de Porto Alegre, the Medical School and the Institute of Psychology of the Universidade Federal do Rio Grande do Sul, done with groups presenting that disease. It starts from the assumption that the study of the relationship between health and work is an important area for the psychologist and that research papers are necessary, based on experience reports, which present, systematize and lay the foundation procedures, has allowed the individuals to decrease his/her culpability and reinforce his/her independence and autonomy, thus minimizing the psychological suffering associated to the chronic pain and limitations imposed by the disease.

Keywords: Repetitive strain injuries; work related illnesses; groups discussion.
\end{abstract}

Nos estudos e práticas sobre as questões relacionadas à saúde e ao trabalho, os aspectos psicossociais vêm, gradualmente, ocupando um espaço crescente pois a presença de uma doença e ou a vivência de um acidente repercutem na vida familiar, laboral e social e no psiquismo do trabalhador. Em geral, acompanhando essas

\footnotetext{
${ }^{1}$ Endereço para correspondência: Rua Ramiro Barcelos, 2600, sala 201, Porto Alegre, RS., 90035-003. Fone (51) 316-5066. E-mail: mjacques@ufrgs.br

${ }^{2}$ Experiência integrante do projeto de pesquisa "Estudo multidisciplinar com trabalhadores portadores de Lesões por Esforços Repetitivos (LER) com dor crônica para a construção de uma nova proposta de tratamento", financiado pelo CNPq. Participam do projeto e colaboraram neste relato: Psicóloga Adriana Silva, Psicóloga Desirée Bianchessi, Psicóloga Márcia Ramos, Psicóloga Sylvia Graciela Sosa Mérola, Médica Jaqueline Elbern, Educadora Física Carla Capozzoli e Educadora Física Regina Albrecht.
}

ocorrências e dependendo da gravidade das mesmas, os indivíduos expressam sentimentos de desvalia, insegurança quanto ao futuro profissional, inconformismo frente a algumas limitações, incerteza e morosidade no processo terapêutico e de reabilitação, medos e fantasias inconscientes, manifestações depressivas e de revolta, associadas, em geral, a incorporação de toda uma ideologia de culpabilização individual.

Algumas patologias relacionadas com o trabalho demandam, necessariamente, propostas de intervenção que vão além dos tratamentos clínico e cirúrgico tradicionais tendo em vista as significativas alterações impostas na vida cotidiana de seus portadores. Uma delas é a LER/DORT (Lesões por Esforços Repetitivos/ Distúrbios Osteomusculares Relacionados ao Trabalho) que apresenta-se como uma das doenças ocupacionais 
mais epidêmicas no Brasil e em outros países (Settimi \& Silvestre, 1995). No Ambulatório de Doenças do Trabalho do Hospital de Clínicas de Porto Alegre (ADTHCPA) representa, hoje, mais de $70 \%$ dos atendimentos.

A recorrência a explicações de ordem subjetiva para a origem da doença e a necessidade de ampliar a conduta terapêutica para além dos tratamentos tradicionais (como o emprego de medicação, fisioterapia, acupuntura e cirurgia) suscitam a inclusão da abordagem psicológica junto a equipes multidisciplinares que atendem trabalhadores com LER/DORT. A partir da descrição clínica da doença e da sua relação com o trabalho, apresenta-se e discute-se neste artigo, proposto como relato de experiência, as contribuições da Psicologia ao estudo da LER/DORT, privilegiando-se a exposição e análise do trabalho com grupos de portadores da doença, prestado por uma equipe multidisciplinar do Hospital de Clínicas de Porto Alegre, em parceria com a Faculdade de Medicina e o Instituto de Psicologia da Universidade Federal do Rio Grande do Sul, além de pesquisadores autônomos.

A proposta deste artigo enquanto relato de experiência e não relato de resultados de pesquisa, justifica-se no pressuposto de que o estudo da relação entre saúde e trabalho constitui-se em um importante espaço de atuação do psicólogo e que são necessárias produções teóricas que apresentem, sistematizem e fundamentem propostas de intervenção. A significatividade das patologias agrupadas como LER/DORT, atingindo cerca de $25 \%$ da população trabalhadora nas estatísticas oficiais e a possibilidade de construção de propostas análogas para outras doenças ocupacionais, ensejam a prioridade conferida a esta patologia (Feuerstein, 1993). Pretendese, com este artigo, apresentar a multiplicação de abordagens para além do tratamento clínico tradicional junto a trabalhadores acometidos de doenças ocupacionais visto a abrangência das implicações de tais quadros patológicos. Neste sentido, prioriza-se a descrição dos procedimentos empregados no trabalho com grupos e não conclusões e resultados como em um relatório de pesquisa. Além disso, o desenvolvimento e a história particular de cada grupo determinam especificidades nos resultados alcançados.

\section{Definição e Fatores de Risco}

Agrupam-se como LER/DORT afecções que podem acometer tendões, sinóvias, músculos, nervos, fácias, ligamentos, de forma isolada ou associada, com ou sem degeneração de tecidos, atingindo, principalmente, mas não tão somente, os membros superiores, região escapular e pescoço, com origem ocupacional. Abrangem quadros clínicos caracterizados pela ocorrência de vários sintomas concomitantes ou não, tais como dor, parestesia, sensação de peso e de fadiga. Entidades neuroortopédicas definidas como tenossinovites, sinovites, compressões de nervos periféricos podem ser identificadas ou não, sendo comum a ocorrência de mais de uma dessas entidades neuro-ortopédicas e a concomitância com quadros mais inespecíficos como a síndrome miofascial (Ministério da Saúde, 1999; Hoefel, 1996). Freqüentemente são causas da incapacidade laboral temporária ou permanente.

Vários fatores associados ao trabalho concorrem para a ocorrência de LER/DORT como a repetitividade de movimentos, a manutenção de posturas inadequadas, o esforço físico, a invariabilidade de tarefas, a pressão mecânica sobre determinados segmentos do corpo, o trabalho muscular estático, impactos e vibrações. A intensificação do ritmo, da jornada e da pressão por produção e a perda acentuada do controle sobre o processo de trabalho por parte dos trabalhadores (fatores relacionados à organização do trabalho), têm sido apontados como os principais determinantes para a disseminação da doença (Assunção \& Rocha, 1995).

A partir de um dos estudos precursores realizado por Kern e Schumann (1984) na Alemanha, muito tem sido falado sobre as transformações no mundo do trabalho. Sem entrar nas extensas polêmicas sobre o nome a darse a essas novas formas de organizar o trabalho, é importante salientar que tais modificações ainda se apresentam no Brasil como um tendência pois o que se encontra são empresas implementando alguns aspectos dessas propostas. Assim, o que se constata, em geral, é o que se poderia chamar de "modelo Frankstein" (Merlo, 1999), onde são incorporados alguns instrumentos usados pelas chamadas "japonizações" da organização do trabalho, tais como Programas de Qualidade Total e Kanban, dentro de políticas de gestão que se mantém verticalizadas, autoritárias e muito hierarquizadas e, em geral, em ambientes insalubres. $O$ que vem se constatando é uma superposição de agressões, umas oriundas das formas tradicionais de gestão, outras impostas pelo processo de reestruturação produtiva.

As evidências epidemiológicas apontam para uma associação de fatores causais interagindo sinergicamente nos processos agudos e na cronificação dessas patologias agrupadas como LER/DORT. Não existe, ainda, conhecimento acumulado que permita quantificar a parcela de cada fator na determinação do esquema global dessas afecções, assim como o evento precipitante de cada caso clínico, visto a intersecção de vários fatores na história de cada trabalhador. E, embora as condições 
objetivas de trabalho sejam explicações consensuais sobre a etiologia dos sintomas, reconhecidas pelos órgãos previdenciários e referidas nas denominações da doença, mantém-se, ainda, a continuidade do debate em torno desta questão.

Chama a atenção pelo peso e interpretação, a recorrência a explicações de ordem psicológica em que a justificativa recai muito menos no ambiente ou na estrutura de trabalho e muito mais em características associadas com fatores de personalidade ou suscetibilidade individual à tensão. Alguns estudos publicados a partir de experiências em atendimento clínico psicoterápico recorrem a explicações fundamentadas em conflitos psicológicos inconscientes com expressão como conversão histérica (Lucire, 1986) e na angústia de castração (Almeida, 1995).

Considerações críticas a este conjunto de explicações não negam a dimensão subjetiva presente na patologia, mas centralizam sua atenção na relação entre o trabalhador (o psicológico e o individual) e o contexto de trabalho. Além disso, a LER/DORT inclui afecções que têm sua origem, não em um agente externo, mas em uma ação, o que pressupõe o envolvimento de um sujeito que a executa. Assim é que, além da auto-identificação dos portadores como pessoas "elétricas", que trabalham em ritmo intenso, ou como "perfeccionistas" e que assumem muitas atividades, é necessário considerar que tais características são sustentadas por uma ideologia de trabalho em que dar o máximo é uma atitude valorizada (Sato e cols., 1993).

\section{O Tratamento da LER/DORT}

Todos os fatores descritos e característicos da LER/ DORT concorrem para seu difícil diagnóstico e tratamento; ainda, seus portadores, em geral, apresentam quadros clínicos onde os sintomas e a dor crônica não condizem com os resultados do exame clínico. Por outro lado, a falta de melhora e a grande incapacidade associada aos casos têm demonstrado a pouca eficácia dos tratamentos isolados.

Como uma proposta terapêutica complementar capaz de considerar a multifatoriedade da LER/DORT, o ADT-HCPA desenvolveu e implantou em sua rotina de atendimento, além dos tratamentos tradicionais, duas modalidades de trabalho com grupos, fundamentadas em experiências similares desenvolvidas por outros pesquisadores (Lima \& Oliveira, 1995; Sato e cols., 1993) e associadas a exercícios de alongamento, relaxamento e fortalecimento muscular.

São objetivos dessas duas modalidades de tratamento: construir alternativas terapêuticas para além da abordagem clínica da LER/DORT; fornecer dados e instrumentalizar os profissionais que trabalham com a doença ao permitir adentrar em dimensões nem sempre visíveis aos observadores externos; desenvolver discussões acerca da doença e da problemática psicossocial que a envolve a fim de minimizar o sofrimento psíquico que lhe é concomitante; construir representações acerca da doença entre seus portadores que miniminizem a culpabilização individual e a desmistificação de explicações baseadas em preconceitos e no senso comum; estimular a multiplicação de conhecimentos construídos através da divulgação de informações; constituir um espaço para que os portadores de LER/DORT possam compartilhar sentimentos e vivências com relação à doença e, assim, criar coletivamente, estratégias de saúde; promover a elaboração de alternativas para lidar com a dor crônica de forma a romper com o ciclo da dor e diminuir as suas repercussões no cotidiano de vida; propor alternativas de trabalho como procedimento terapêutico, respeitando a capacidade residual de cada portador; propiciar o aumento da flexibilidade e fortalecimento muscular com orientações auto-aplicáveis de exercícios físicos; e. avaliar a eficácia da associação de esquemas terapêuticos conservadores (medicação, fisioterapia, acupuntura) com o tratamento proposto. Estimula-se, entre os participantes, a utilização de estratégias individuais e ou coletivas de convivência com os limites impostos pela doença como propostas de ajuda mútua geridas pelos próprios portadores em outros espaços da comunidade (Centros de Referência, Sindicatos, ONGs, etc.), ações individuais e ou coletivas de (re)inserção em atividades diversas, inclusive de caráter produtivo e o aumento da amplitude articular. A amplitude dos objetivos propostos não implica em desconsiderar as diferenças de grupos e de indivíduos em particular, o que, em muitos casos, limita tais objetivos.

As modalidades de tratamento com grupos foram denominadas de Grupos Temáticos e Grupos de Intervenção. Os primeiros têm como característica serem previamente estruturados, de curta duração, com objetivos operacionais definidos, servirem de introdução prévia para outras abordagens grupais e abrangerem um número extensivo de portadores de LER/DORT. Os segundos, de acordo com a rotina proposta, devem ocorrer posteriormente à realização de Grupos Temáticos pois sua eficácia tende a aumentar se já tiver sido previamente trabalhada a participação em grupos de discussão. A avaliação desses grupos é realizada através de uma abordagem objetiva (exame da incapacidade física) e de uma apreciação dos participantes quanto às expectativas alcançadas. 
Sinteticamente, o fluxo de atendimento dos portadores de LER/DORT ficou assim estabelecido:

$1^{\circ}$ nível: Diagnóstico através da história ocupacional, exames clínico e/ou laboratoriais e estabelecimento do grau de incapacidade;

$2^{\circ}$ nível: Grupos Temáticos associados a exercícios físicos;

$3^{\circ}$ nível: Grupos de Intervenção associados a exercícios físicos;

Avaliação clínica periódica.

\section{Procedimentos}

Grupos Temáticos: Enquadre

Desenvolvem-se através de seis reuniões semanais, com uma hora e meia de duração (incluindo meia hora de exercícios físicos), com oito a dez participantes e uma equipe técnica constituída de três profissionais: psicólogo e médico, que se alternam nos papéis de coordenador e co-coordenador e um profissional de educação física. Os encontros são estruturados a partir de temas previamente definidos e são utilizadas técnicas grupais para facilitar a emergência dos conteúdos a serem trabalhados.

Após um primeiro encontro de apresentação da proposta e do levantamento das expectativas, os dois encontros seguintes são dedicados à discussão das características dos trabalhos executados pelos portadores de LER/DORT e suas relações com tal patologia. Os participantes discorrem sobre aspectos como ritmo, jornada, equipamentos utilizados, hierarquia e controle experienciados nos locais de trabalho, incluindo, em alguns casos, dramatizações de situações do cotidiano laboral e resolução de dúvidas acerca da doença. Estimula-se a tomada de consciência sobre as formas como se organizam os contextos de trabalho onde cada indivíduo se insere, a atenção às regularidades entre estes contextos e a relação com as causalidades da LER/DORT, favorecendo a construção de representações sobre o papel do trabalho na etiologia da doença.

Os dois encontros seguintes abordam os sentimentos e vivências decorrentes do convívio com os sintomas e suas repercussões no cotidiano de vida, bem como a construção de estratégias individuais e coletivas para enfrentar as limitações que lhe são coexistentes. Através da seleção, recorte e colagem de imagens em um cartaz coletivo, são estimuladas as manifestações de emoções, frustrações, expectativas, representações e ansiedades que acompanham o convívio com a doença e a socialização de práticas individuais e coletivas que auxiliam na adaptação ao cotidiano de vida.

O último encontro é de avaliação, quando são retomadas as expectativas apuradas na primeira reunião.
Confronta-se a apreciação dos participantes com tais expectativas, avalia-se os resultados alcançados e propõese a continuidade do trabalho em uma nova modalidade de intervenção.

Os fundamentos teóricos baseiam-se na experiência desenvolvida em São Paulo desde 1992 (Sato e cols., 1993), na qual é conferida importância ao espaço grupal como um apoio social na tomada de consciência sobre o papel dos contextos de trabalho na etiologia da LER/ DORT e na construção de um saber coletivo que sirva como instrumentalização para enfrentar a culpa decorrente e a realidade de convívio com os limites impostos pelos sintomas.

\section{Grupos de Intervenção: Enquadre}

Realizam-se reuniões semanais de uma hora e meia de duração. O tempo grupal é subdividido em: sessenta minutos de discussão grupal e trinta minutos de exercícios físicos. O contrato inicial é de dez encontros, com a possibilidade de ser renovado. O primeiro encontro é estruturado, quando se estabelece o contrato de trabalho, expõe-se a proposta de tratamento, seus objetivos, diferenças com o Grupo Temático, número de encontros, duração, freqüência e comprometimento com a assiduidade e o sigilo.

No segundo encontro o grupo faz atividades com técnicas de colagem para tentar expressar o que foi compreendido da proposta apresentada no encontro anterior, com ênfase no exame das estratégias a serem alcançadas no final do trabalho proposto. Os encontros posteriores são abertos, trabalhando-se a partir dos emergentes grupais, como também, de alguma proposta ou tema avaliado como pertinente. A equipe de coordenação é composta por psicólogo, médico e profissional de educação física.

Estimula-se que o portador de LER/DORT (re)aprenda a utilizar de seu potencial criativo para construir estratégias de saúde ao lidar com a dor crônica. Essas estratégias se expressam em alternativas de independência, rompendo a incapacidade manifesta nas relações familiares, no trabalho, no atendimento médico, nas perícias na Previdência Social. Este processo permite (re)apropriar-se de elementos subjetivos que favorecem a diminuição da contratura e da dor.

Os fundamentos teóricos utilizados são o do grupo operativo (Pichón, 1988) e o do grupo enquanto dispositivo (Barros, 1997). A proposta estabelecida coletivamente pelo grupo implica um fazer, que é refletir criticamente sobre si mesmo e sobre as relações que vão se estabelecendo em função do objetivo proposto. Esta reflexão é básica para visualizar e resolver os obstáculos 
frente às mudanças, cuidar das ansiedades emergentes em relação ao que é proposto e elaborar outras ansiedades, medos e perdas associadas.

Os mecanismos de saúde são construídos com o novo conhecimento decorrente de um conjunto de experiências e de afetos que são mobilizados pelos integrantes do grupo ao pensarem, sentirem, agirem, tanto individual como coletivamente, sobre a tarefa. Nesse processo de aprendizagem é gerada mudanças na realidade e no próprio indivíduo, tornando possível a ruptura que as obstaculizam.

Após cada sessão de grupo são realizados exercícios de relaxamento, alongamento e fortalecimento muscular. Similarmente ao que ocorre nos Grupos Temáticos, propõe-se uma reeducação postural e de reconhecimento dos próprios limites físicos, destacando a importância da atividade e o comprometimento com uma rotina diária de exercícios físicos. Procura-se, paulatinamente, aumentar a flexibilidade, a resistência e permitir movimentos sem dor, buscando uma melhora da capacidade funcional e residual e do quadro álgico.

\section{Considerações Finais}

Os grupos de discussão e reflexão com portadores de LER/DORT, nas duas modalidades apresentadas anteriormente, vêm se constituindo em uma alternativa complementar no tratamento desta patologia. Os Grupos Temáticos permitem a participação de um maior número de trabalhadores por preverem um número fixo e reduzido de encontros; funcionam, também, como sensibilizadores pois fornecem um modelo de intervenção que rompe a relação dual médico-paciente e a crença nessa relação como a única alternativa de tratamento. Os Grupos de Intervenção permitem a abordagem das problemáticas emergentes, respeitando os limites e especificidades de cada grupo. A inclusão de exercícios físicos como integrantes da atividade proposta, além dos benefícios que lhes são inerentes, estimula a participação discursiva, a coesão grupal e diminui a ansiedade frente a um modelo de intervenção que, em geral, esses trabalhadores estão pouco familiarizados.

Os portadores de LER/DORT abrangidos pelo atendimento do ADT-HCPA são pacientes do Sistema Único de Saúde (SUS), oriundos, principalmente, dos setores bancário, metalúrgico e calçadista, e, em geral, estão afastados temporariamente do trabalho. Dos participantes nos grupos já realizados, $82 \%$ eram do sexo feminino, $84 \%$ tinham entre 31 e 50 anos, $47 \%$ com primeiro grau incompleto, $80 \%$ com filhos, $43 \%$ recebendo até três salários mínimos, $84 \%$ com jornada de oito horas semanais, $62 \%$ com hábito de horas-extras até três vezes por semana, 70\% utilizando força física no exercício laboral, $80 \%$ com braço sem apoio na execução das tarefas, $69 \%$ sem possibilidade de planejamento do processo de trabalho e $64 \%$ com exigência de produtividade controlada pela chefia. Tais características demográficas e ocupacionais apresentadas são semelhantes àquelas que as estatísticas apresentam como típicas dos trabalhadores com diagnóstico de LER/ DORT (Assunção \& Rocha, 1995; Settimi \& Silvestre, 1995) o que permite generalizar a proposta a outros grupos acometidos com tal patologia.

Tem-se constatado uma aceitabilidade do trabalho entre os participantes que se expressa pelo baixo absenteísmo às reuniões e pelo desejo manifesto de se engajar em diferentes propostas de trabalho em grupo. A avaliação clínica não revela uma melhora na totalidade dos pacientes a não ser uma menor freqüência na agudização dos sintomas , tendo em vista, principalmente, o melhor auto-controle na realização de algumas atividades, especialmente as atividades domésticas.

Uma conduta freqüente entre portadores de LER/ DORT é a dependência, passividade e resignação, além de constantes manifestações queixosas; revelam expectativas de que exista uma solução externa capaz de salvar-lhes da doença, da incapacidade e da dor crônica. São justamente tais características que o trabalho com grupos procura minimizar. Utilizando-se de cenas da incapacidade que se expressam nas relações sociais, são estimuladas atitudes e comportamentos de independência e auto-controle, como procurar fazer sempre que possível as atividades sem ajuda e aprender a pedi-la quando tal atividade demanda um esforço que agrava a sintomatologia.

Uma característica muito presente nas reuniões é a discussão sobre como tornar o trabalho uma alternativa terapêutica, através de terapias ocupacionais ou de retorno a alguma atividade produtiva. A pouca qualificação das pessoas envolvidas dificulta o processo de escolha. A busca por equipamentos que facilitem o exercício de atividades manuais e a proposta de buscar orientação profissional especializada são algumas das alternativas escolhidas. As reflexões nos grupos e as experiências dos diferentes membros que o constituem, mostram-se como estímulos encorajadores para a busca de um fazer em acordo com as limitações impostas pela doença. A importância da ação/reflexão e do desenvolvimento de conhecimentos como forma de inserção no mundo, apontada exaustivamente na literatura sobre grupos, tem sido o eixo condutor do trabalho desenvolvido. No entanto, não são raras as situações individuais de testar a capacidade de realizar algumas tarefas, o que, em geral, 
provoca agudização dos sintomas e frustração. $\mathrm{O}$ exame dessas situações tem sido uma proposta constante, sensibilizando para o uso do auto-controle e do reforço grupal.

Considera-se que a utilização de trabalhos com grupos tem possibilitado que o portador de LER/DORT possa (re)aprender a utilizar seu potencial na busca de recursos para construir estratégias de saúde e, assim, lidar de forma mais autônoma com a dor crônica. Constatou-se maiores mudanças na relação com a forma de trabalhar e exercer atividades domésticas, empregando a adaptação de objetos, a solicitação de auxílios e uma maior aceitação e respeito aos limites impostos pela doença. Quase todos os participantes passaram a usar algum dispositivo para evitar a dor, em especial, com relação à indução ao sono. Também, constatou-se mudanças, embora em menor número de indivíduos, nas relações familiares (busca de diálogo com a família), no apetite, no lazer (busca de novas formas de lazer, evitando alguns tipos de programas), na higiene (usar novas estratégias, buscar a ajuda de outra pessoa) e na vida sexual. Embora nem todos os participantes alcancem o mesmo nível de engajamento e o emprego de estratégias que miniminizem o sofrimento advindo da doença, a diminuição da culpabilização tem sido observado como positivo, aliviando o sofrimento manifesto associado a patologia.

A inclusão da proposta de trabalho com grupos como tratamento complementar tem se mostrado mais abrangente (para além da esfera clínica tão somente) do que o trabalho tradicional realizado por apenas uma especialidade e assim, capaz de satisfazer a complementaridade que é exigida pelas características da própria patologia e das doenças ocupacionais em geral. Tem se mostrado, também, como um importante instrumento de visualização para os técnicos de dimensões da LER/DORT não restritas ao aspecto clínico. No entanto, não substitui outras estratégias de maior abrangência que se centralizam nas políticas públicas e nos ambientes de trabalho e que sejam capazes de prevenir a incidência alarmante de casos de LER/DORT, particularmente em países com as características do Brasil.

\section{Referências}

Almeida, M.C. (1995). Características emocionais determinantes da LER. Em W. Codo \& M.C. Almeida (Orgs.), LER (pp. 24-56). Petrópolis, RJ: Vozes.

Assunção, A . \& Rocha, L. (1995). Agora... até namorar fica difícil: Uma história de lesões por esforços repetitivos. Em J.T. Buschinelli, L. Rocha \& R. Rigotto (Orgs.), Isto é trabalho de gente? (pp. 461-473). Petrópolis, RJ: Vozes.

Barros, R.B. de (1997). Dispositivos em ação: O grupo. Saúdeloucura, 6, $183-$ 191.

Feuerstein, M. (1993). Multidisciplinary rehabilitation of chronic workrelated upper extremity disorders. Journal of Occupational Medicine, 35(4), 396-403.

Hoefel, M.G. L. (1996). Lesões por esforços repetitivos. Em B. Duncan, M.I. Schmidt \& E. Giuliani (Orgs.), Medicina ambulatorial: Condutas clínicas em atenção primária (pp. 762-746). Porto Alegre: Artes Médicas.

Kern, H. \& Schumann, M. (1989). La fin de la division du travail? La rationalisation dans la production industrielle. Paris: Maison des Sciences de l'Homme.

Lima, A. \& Oliveira, F. (1995). Abordagem psicossocial da LER: Ideologia da culpabilização e grupos de qualidade de vida. Em W. Codo \& M. C. Almeida (Orgs.), LER (pp. 136-159). Petrópolis, RJ: Vozes.

Lucire, Y. (1986). Neurosis in the workplace. The Medical Journal of Australia, 145, 323-327.

Merlo, A .R. C. (1999). A informática no Brasil: Prazer e sofrimento no trabalbo. Porto Alegre: Editora da Universidade/UFRGS.

Ministério da Saúde. Comitê Assessor das LER/DORT. (1999). Protocolo de investigação, diagnóstico, tratamento e prevenção de LER/DORT. Brasília.

Pichón, E. (1988). O processo grupal. São Paulo: Martins Fontes.

Sato, L., Araújo, M., Udihara, M. L., Nicotera, F., Dalton, M.T., Settimi, M. \& Silvestre, M. (1993). Atividades em grupo com portadores de LER e achados sobre a dimensão psicossocial. Revista Brasileira de Saúde Ocupacional, 79(21), 49-62.

Settimi, M. \& Silvestre, M. (1995). Lesões por esforços repetitivos (LER): Um problema da sociedade brasileira. Em W. Codo \& M.C. Almeida (Orgs.), LER (pp. 321-355). Petrópolis, RJ: Vozes.

Sobre os autores:

Álvaro Roberto Crespo Merlo é professor da Universidade Federal do Rio Grande do Sul.

Maria da Graça Corrêa Jacques é professor da Universidade Federal do Rio Grande do Sul.

Maria da Graça Luderitz Hoefel trabalha no Hospital de Clínicas de Porto Alegre.

Recebido em 21/09/2000

Revisado em 16/10/2000

Aceito em 5/12/2000 